\title{
Association between Serum Cytokeratin-18 Neoepitope M30 (CK-18 M30) Levels and Chronic Hepatitis B: A Meta-Analysis
}

\author{
Changfei Li, ${ }^{1}$ Shousheng Liu, ${ }^{2,3}$ Linlin Lu, ${ }^{2,3}$ Quanjiang Dong, ${ }^{3,4}$ Shiying Xuan, ${ }^{1,2,4,}$ and Yongning \\ $\mathrm{Xin}^{1,2,4,5, * *}$ \\ ${ }^{1}$ Medical College of Qingdao University, Qingdao 266071, China \\ ${ }^{2}$ Digestive Disease Key Laboratory of Qingdao, Qingdao 266071, China \\ ${ }^{3}$ Central Laboratories, Qingdao Municipal Hospital, Qingdao 266071, China \\ ${ }^{4}$ Department of Gastroenterology, Qingdao Municipal Hospital, Qingdao, Shandong Province, China \\ ${ }^{5}$ Department of Infectious Disease, Qingdao Municipal Hospital, Qingdao 266011, China \\ "Corresponding author: Shiying Xuan, Department of Gastroenterology, Qingdao Municipal Hospital, 1 Jiaozhou Road, Qingdao 266011, Shandong Province, China. Tel: \\ +86-53288905508, Fax: +86-53288905293, E-mail: xuansydxy@163.com \\ "Corresponding author: Yongning Xin, Department of Gastroenterology, Qingdao Municipal Hospital, 1 Jiaozhou Road, Qingdao 266011, Shandong Province, China. Tel: \\ +86-53282789463, Fax: +86-53285968434, E-mail: xinyongning@163.com
}

Received 2017 November 17; Accepted 2018 April 20.

\begin{abstract}
Background: Cytokeratin-18 Neoepitope M30 (CK-18 M30) has been reported to be associated with chronic HBV infection and severity of liver injury; however, the results of these studies are inconsistent.

Objectives: We sought to investigate the association between serum CK-18 M30 levels and the severity of liver injury in chronic hepatitis $\mathrm{B}(\mathrm{CHB})$ patients.

Methods: A systematic literature search was performed in PubMed, Embase, and Cochrane Library databases for relevant studies published in English up to August 2017. Heterogeneity among individual studies was investigated for summarizing all the studies. The standard mean difference (SMD) and 95\% confidence interval (CI) were calculated using a random-effects model or fixed-effects model. Finally, the sensitivity analysis and publication bias were performed to evaluate the accuracy of this meta-analysis. Statistical analysis was conducted using Review Manager 5.3 and Stata 12.0.

Results: Five case-control studies were included in the ultimate analysis, recruiting 488 CHB patients, 276 inactive carriers, and 193 healthy controls. The major results of the meta-analysis revealed significantly elevated serum CK-18 M30 levels in chronic HBV infected patients including CHB patients with severe liver injury and inactive HBV carriers when compared to healthy controls (SMD $=1.13,95 \%$ CI: $0.75-1.50, \mathrm{P}<0.001$; SMD $=0.63,95 \%$ CI: $0.38-0.89, \mathrm{P}<0.001$, respectively). Furthermore, the serum CK-18 M30 levels were significantly higher in CHB patients with severe liver injury than in inactive carriers (SMD =1.29, 95\% CI: $0.60-1.98, \mathrm{P}<0.001$ ). The sensitivity and publication bias analysis verified the stability and reliability of our analysis.

Conclusions: The elevated serum CK-18 M30 levels could be regarded as a useful non-invasive biomarker for the diagnosis of chronic HBV infection, and were associated with the severity of liver injury in chronic Hepatitis B patients. The serum CK-18 M30 levels could reflect the liver inflammation in inactive carriers, representing the early stage of chronic HBV infection.
\end{abstract}

Keywords: Cytokeratin-18 M30, Chronic Hepatitis B, Liver Injury, Meta-Analysis

\section{Background}

Hepatitis B virus (HBV) is the prototype of the Hepadnaviridae (hepatotropic DNA virus) family and has a strong preference for infecting liver cells (1). HBV infection is a major global health problem with approximately 350 million people chronically infected worldwide, especially in Asia, accounting for a large morbidity and mortality of liver disease (2-4). HBV infection leads to the liver injury and inflammation and gradually progresses to liver fibrosis, cirrhosis, and even hepatocellular carcinoma (HCC) (5,
6). The spectrum of the disease and the history of chronic $\mathrm{HBV}$ infection are various and variable, ranging from inactive carrier state to progressive chronic hepatitis $\mathrm{B}(\mathrm{CHB})$ (7). The primary chronic HBV infection in adults and children can be either symptomatic or asymptomatic, and the long-term HBV infection increases the risk of developing fibrosis and HCC (8). Therefore, it is very important and necessary to diagnose the liver injury precisely during the chronic HBV infection in the early stage and provide a reasonable treatment scheme to prevent the progression of liver disease. 
Up to now, the liver biopsy is still considered as the gold standard for the staging of hepatic inflammation and fibrosis. The application of liver biopsy is limited because of its high cost, invasiveness, and the association with the risk of complications. Furthermore, the diagnostic accuracy of liver biopsy decreases due to sampling errors and a significant rate of intra- and inter-observer variability (911). Currently, some novel non-invasive methods are widely used to assess the severity of liver injury, including serum markers, such as alanine transaminase (ALT) (12), aspartate aminotransferase (AST) (13), Cytokeratin-18 (CK-18) (14), and aspartate aminotransferase to platelet ratio index (APRI) (13), and imaging techniques such as transient elastography (TE) (15) and magnetic resonance elastography (MRE) (16).

Cytokeratin-18 (CK-18) is a main cytoskeletal protein in hepatocytes and other epithelial cells. CK-18 could be released into serum by the dying cells as a cell death signal (17). Two forms of CK-18 are observed in the serum including the whole CK-18 protein (CK-18 M65) and the caspase 3cleaved fragment (CK-18 M30). CK-18 M65 is used to evaluate the total cell death whereas CK-18 M30 is specifically used to evaluate the apoptotic-associated cell death (18). Accumulated evidence suggests that hepatocyte apoptosis plays a vital role in chronic liver disorders $(17,19)$. Yang et al. found that the serum CK-18 levels might be a risk factor for non-alcoholic steatohepatitis (NASH), chronic hepatitis $\mathrm{C}(\mathrm{CHC})$, and chronic hepatitis $\mathrm{B}(\mathrm{CHB})($ all $\mathrm{P}<0.05)$ through conducting a meta-analysis of eight case-control studies (20). Some reports also showed that the plasma levels of CK-18 M30 were associated with the progression of non-alcoholic steatohepatitis (NASH) in non-alcoholic fatty liver disease (NAFLD) patients $(21,22)$. Recently, the associations between serum levels of CK-18 M30 and the progression of the chronic HBV infections were investigated. However, the results have been highly variable. Therefore, we performed a meta-analysis to explore the association between serum CK-18 M30 levels and chronic HBV infection and the progression of chronic hepatitis $\mathrm{B}$.

\section{Objectives}

The aim of this study was to investigate the association between serum CK-18 M30 levels and the severity of liver injury in chronic hepatitis $\mathrm{B}(\mathrm{CHB})$ patients.

\section{Methods}

\subsection{Search Strategy}

A systematic literature search was performed in PubMed, Embase, and Cochrane Library for relevant studies about humans published in English up to August 2017.
The following keywords were used during the search: "HBV infection" or "chronic virus hepatitis" or "chronic hepatitis B" or "CHB" and "Cytokeratin-18" or "Keratin-18" or "CK-18" or "CK18." Studies that were not published as full reports, such as conference abstracts, letters, and case reports were excluded. In addition, a manual literature search was conducted using the references of original manuscripts and reviews to identify additional related studies. We also contacted the authors of studies containing relevant information, who did not report the results necessary for this analysis. The eligible reports were identified by two investigators (CF Li and SS Liu) independently, and controversial studies were resolved through iteration and consensus.

\subsection{Study Selection}

The study eligibility was also independently determined by the same two investigators (CF Li and SS Liu), and any disagreement was resolved by consensus. Studies meeting the following inclusion criteria were included: (I) studies evaluating the association between serum CK-18 M30 levels and chronic hepatitis B; (II) studies on chronic hepatitis $B$ patients who possessed features including positivity for HBsAg, $>10^{5}$ copies per $\mathrm{mL}$ of serum HBV DNA, and/or elevated ALT levels (more than the upper limit of normal on $>2$ separate monthly decision within the last 6 months); (III) articles published with full text and; (IV) studies with sufficient information on the serum CK-18 M30 levels in the cases and controls. The criteria for exclusion were as follows: (I) studies not satisfying the inclusion criteria; (II) letters, case report, conference abstracts, reviews, or meta-analyses; (III) repeated publications or studies with over-lapping data; (IV) studies without control subjects or studies that involved less than 30 participants; (V) studies that included patients co-infected with NAFLD or HCV or acute hepatitis, having alcohol-related liver diseases, or having received antiviral treatments previously.

\subsection{Data Extraction and Quality Assessment}

Two investigators (CF Li and SS Liu) independently extracted data from eligible studies. Disagreements were resolved by discussion and consensus. For each study, the following information was extracted from each eligible study: the name of the first author, the year of publication, the place of study conduction, the patient age, gender, publication language, the number of cases and controls, serum CK-18 M30 levels in the case and control group, and the detection method of CK-18 M30 in each study. The Newcastle-Ottawa Scale (NOS) was used to evaluate the quality of studies enrolled in our meta-analysis (23). The 
NOS is composed of eight questions with nine possible points that included three aspects: (I) subjects selection: 0 - 4; (II) comparability in subjects: 0 - 2 and; (III) ascertainment for the exposure: 0 - 3. In this meta-analysis, studies receiving 6 or higher scores were regarded as high-quality studies. The heterogeneity test was conducted among these studies using Q-test $(\mathrm{P}<0.05$ was regarded as statistically significant) and $\mathrm{I}^{2}$ index. A value of $25 \%-50 \%$ for $\mathrm{I}^{2}$ indicates a low degree of heterogeneity, a value of $50 \%$ - 75\% indicates a moderate heterogeneity, and a value of $>75 \%$ indicates a high heterogeneity. When the $\mathrm{I}^{2}$ value was $>50 \%$, a random-effects model was used to pool the data; otherwise, a fixed-effects model was selected (20). The association between serum CK-18 M30 levels and CHB was estimated by the standard mean difference(SMD) with 95\% confidence interval (CI). Sensitivity analysis was performed to investigate the source of heterogeneity by removing a study each time to assess the stability of our results. $\mathrm{P}<0.05$ was considered statistically difference. The Funnel plot was used to evaluate the possible publication bias using the Begg's test; $\mathrm{P}<0.05$ represented the significant asymmetry of the funnel plot. All analyses were performed using Review Manager 5.3 (Cochrane Collaboration, Oxford, UK) and Stata 12.0 (Stata Corporation, Texas, US).

\section{Results}

\subsection{Literature Search}

A total of 136 articles were retrieved initially through electronic database searching and manual search. 34 papers were kept after removal of duplicates $(n=33)$, reviews, letters or case reports $(n=12)$, unpublished passages or conference abstracts $(n=5)$, studies with other diseases ( $n$ $=37$ ), and studies unrelated to the research topics $(n=15)$. Furthermore, 29 additional studies were excluded because they were animal or in-vitro studies $(n=5)$, coincident with other diseases $(n=15)$, insufficient data $(n=7)$, or without control groups $(n=2)$. Eventually, 5 reports were included in the meta-analysis. Figure 1 shows the flowchart of the literature search conducted in this study.

\subsection{Characteristics of Included Studies}

Five studies published from 2008 to 2017 were eligible for meta-analysis (17, 24-27). The basic features of these studies are shown in Table 1 . Four studies were performed in populations of Turkey and one study was performed in populations of Greece. A total of $488 \mathrm{CHB}$ patients, 276 inactive carriers, and 193 healthy controls was included in this study. All the studies were case-control trials; patients in two studies were all negative for $\operatorname{HBeAg}(24,25)$, and one

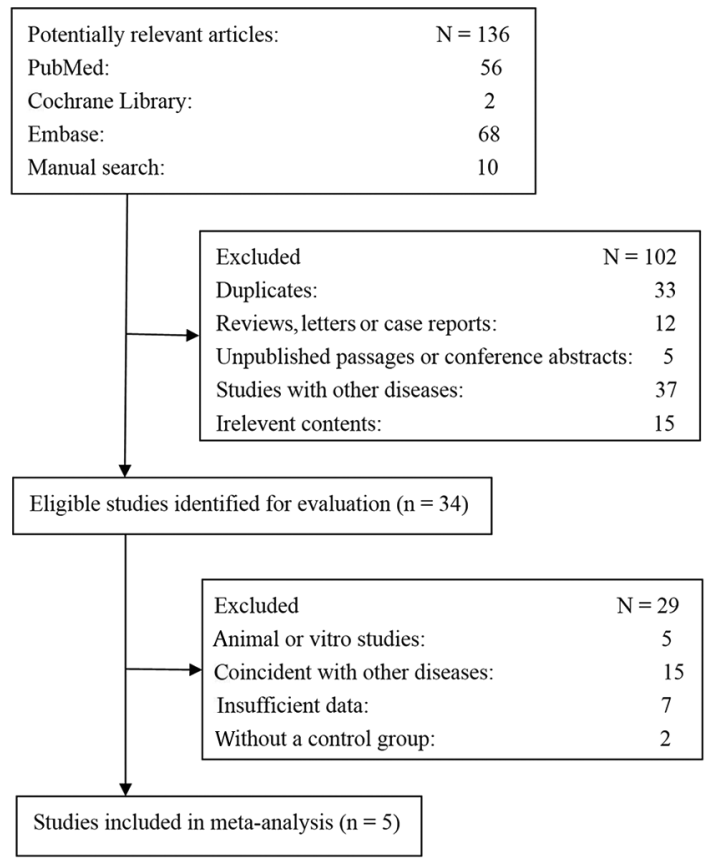

Figure 1. Flowchart of the literature search resulting in 5 included studies. Flow diagram showing the review process and the number of included and excluded studies

study divided the $\mathrm{CHB}$ patients into HBeAg positive and HBeAg negative (17). All the CHB patients were diagnosed by liver biopsy, and the immunohistochemistry (IHC) or ELISA methods were used to test the serum CK-18 M30 levels in the five studies. The results of methodological quality assessment using NOS and the scores of the included articles were between 6 and 7, representing the high quality of studies.

\subsection{Association of the Serum CK-18 M30 Levels with Chronic HBV} Infection

Heterogeneity analysis results $\left(\mathrm{I}^{2}>50 \%\right)$ showed that there was a significant heterogeneity among the studies; thus, the random-effects model was used. As shown in Figure 2, the elevated serum CK-18 M30 levels were observed in chronic HBV infected patients compared to healthy controls (SMD $=0.95,95 \% \mathrm{CI}: 0.66-1.24, \mathrm{P}<0.001)$ in this metaanalysis. Based on the phase and the severity of chronic HBV infections, we divided the patients into two subgroups: CHB patients with severe liver injury group and inactive carriers group. The subgroup analysis revealed that serum CK-18 M30 levels were significantly higher in CHB patients with severe liver injury and inactive carriers compared to healthy controls (SMD = 1.13, 95\% CI: $0.75-1.50, \mathrm{P}$ 


\begin{tabular}{|c|c|c|c|c|c|c|c|c|c|c|c|c|c|c|c|}
\hline \multirow[t]{2}{*}{ First Author } & \multirow[t]{2}{*}{ Year } & \multirow[t]{2}{*}{ Country } & \multirow[t]{2}{*}{ Total } & \multicolumn{3}{|c|}{ Sample Size } & \multicolumn{3}{|c|}{ Gender, $M / F$} & \multicolumn{3}{|c|}{ Age, $y$} & \multirow{2}{*}{$\begin{array}{l}\text { Diagnose } \\
\text { Crite- } \\
\text { ria }\end{array}$} & \multirow{2}{*}{$\begin{array}{l}\text { Method } \\
\text { (CK 18 } \\
\text { M30) }\end{array}$} & \multirow[t]{2}{*}{ NOS } \\
\hline & & & & CHB & $\begin{array}{l}\text { Inactive } \\
\text { Carri- } \\
\text { ers }\end{array}$ & Controls & CHB & $\begin{array}{l}\text { Inactive } \\
\text { Carri- } \\
\text { ers }\end{array}$ & Controls & CHB & $\begin{array}{l}\text { Inactive } \\
\text { Carriers }\end{array}$ & Controls & & & \\
\hline Papatheodoridis & 2008 & Greece & 145 & $62(-)$ & 53 & 30 & $45 / 17$ & $30 / 23$ & $\mathrm{NA}$ & $48 \pm 15$ & $41 \pm 13$ & $\mathrm{NA}$ & $\begin{array}{c}\text { Liver } \\
\text { biopsy }\end{array}$ & $\mathrm{IHC}$ & 7 \\
\hline Eren & 2010 & Turkey & 172 & $\begin{array}{l}42(+), \\
47(-)\end{array}$ & 54 & 29 & $\begin{array}{l}21 / 26, \\
22 / 20\end{array}$ & $28 / 26$ & $17 / 12$ & $\begin{array}{l}45.9 \pm 9.7 \\
47.2 \pm 8.5\end{array}$ & $44.7 \pm 9.9$ & $46.6 \pm 9.3$ & $\begin{array}{l}\text { Liver } \\
\text { biopsy }\end{array}$ & ELISA & 6 \\
\hline Sumer & 2013 & Turkey & 240 & $189(-)$ & 1 & 51 & $132 / 57$ & 1 & $25 / 26$ & $37.8 \pm 12.6$ & 1 & $33.8 \pm 9.3$ & $\begin{array}{c}\text { Liver } \\
\text { biopsy }\end{array}$ & ELISA & 7 \\
\hline Yilmaz & 2016 & Turkey & 88 & 48 & 1 & 40 & $25 / 23$ & 1 & $14 / 26$ & $45.66 \pm 11.45$ & 1 & $41.67 \pm 13.68$ & $\begin{array}{l}\text { Liver } \\
\text { biopsy }\end{array}$ & ELISA & 7 \\
\hline Balkan & 2017 & Turkey & 312 & 100 & 169 & 43 & $63 / 37$ & $94 / 75$ & $31 / 12$ & $33.54 \pm 11.74$ & $36.89 \pm 10.97$ & $31.28 \pm 7.49$ & $\begin{array}{l}\text { Liver } \\
\text { biopsy }\end{array}$ & ELISA & 7 \\
\hline
\end{tabular}

Abbreviations: CHB, chronic hepatitis B; ELISA, enzyme linked immunosorbent assay; F, Female; IHC, Immunohistochemistry; M, Male; NA, not available; NOS, Newcastle-Ottawa Scale; (+), HBeAg positive; (-), HBeAg negative.

$<0.001 ;$ SMD $=0.63,95 \%$ CI: $0.38-0.89, \mathrm{P}<0.001$, respectively) (Figure 2).

\subsection{Serum CK-18 M30 Levels between CHB Patients and Inactive Carriers}

The differences of serum CK-18 M30 levels between CHB patients with severe liver injury and inactive carriers were investigated. A random-effects model was used because a heterogeneity was existed $\left(\mathrm{I}^{2}>50 \%\right)$. The results showed that $\mathrm{CHB}$ patients with severe liver injury had higher serum CK-18 M30 levels than inactive carriers in this meta-analysis (SMD = 1.29, 95\%CI: 0.60 - 1.98, P < 0.001) (Figure 3).

\subsection{Sensitivity Analysis and Publication Bias}

Sensitivity analysis was performed to evaluate the robustness of the meta-analysis. The removal of any single study did not change the overall statistical difference, suggesting that the current meta-analysis data of each group were relatively stable and reliable. Begg's tests were performed for the publication bias analysis of the groups between chronic HBV infected patients and healthy controls, inactive carriers and healthy controls, and $\mathrm{CHB}$ patients and inactive parries. The results showed that no significant publication bias was existed among the studies $(\mathrm{P}=0.452$; $\mathrm{P}=1.000 ; \mathrm{P}=0.089$, respectively) (Figure 4 ).

\section{Discussion}

Cytokeratin-18 is as a cytoskeletal protein found mainly in epithelial cells and other epithelial cells plays a key role in maintaining the shape of cells and resisting mechanical stresses (28). Previous research reported that CK-18 is expressed by most types of carcinoma cells, such as breast, prostate, lung, liver, colon, and ovary cells, and could be regarded as a useful serum marker for evaluating the clinical progression of patients with epithelial malignant tumors $(29,30)$. Recently, the increased serum levels of CK18 in severe liver diseases were observed suggesting that it may be as a potential serum marker for the diagnosis of clinical liver disease. CK-18 is composed of two molecular forms: the whole CK-18 protein (CK-18 M65) and the caspase-cleaved fragment (CK-18 M30), both of which can be detected in the serum. Accumulated evidence showed that the levels of CK-18 M30 are significantly associated with NASH progression in NAFLD patients of both adults and children $(14,31,32)$. Chen et al. reported that the sensitivity and specificity of CK-18 M30 for NASH were 83\% and $71 \%$ via a meta-analysis of nine studies (33).

The association of serum CK-18 M30 levels with the chronic hepatitis B has well been studied (17, 24-27). This comprehensive meta-analysis investigated the relationship of serum CK18 M30 levels with chronic HBV infection. As shown in the results, the serum CK-18 M30 levels were significantly higher in $\mathrm{CHB}$ patients with severe liver injury than in healthy controls (SMD = 1.13, 95\% CI: $0.75-1.50, \mathrm{P}<$ 0.001). Swiderska et al. reported that the serum CK-18 M30 levels were sensitive and specific for the discrimination of mild, moderate, or severe fibrosis and inflammation even in patients with normal ALT activity (34). In order to investigate the relationship of serum CK-18 M30 levels with degree of the chronic HBV infection, we paid attention to the serum CK-18 M30 levels in the CHB patients with severe liver injury and inactive carriers in this meta-analysis. A significantly higher serum CK-18 M30 level was observed in $\mathrm{CHB}$ patients with severe liver injury compared to inactive carriers (SMD $=1.29,95 \% \mathrm{CI}$ : $0.60-1.98, \mathrm{P}<0.001)$. Sensitivity and publication bias analysis verified the accuracy of this analysis. These results suggest that the serum CK18 M30 levels are distinguished in the CHB patients with severe liver injury compared to the inactive carriers and healthy controls. Serum CK-18 M30 levels that are deter- 


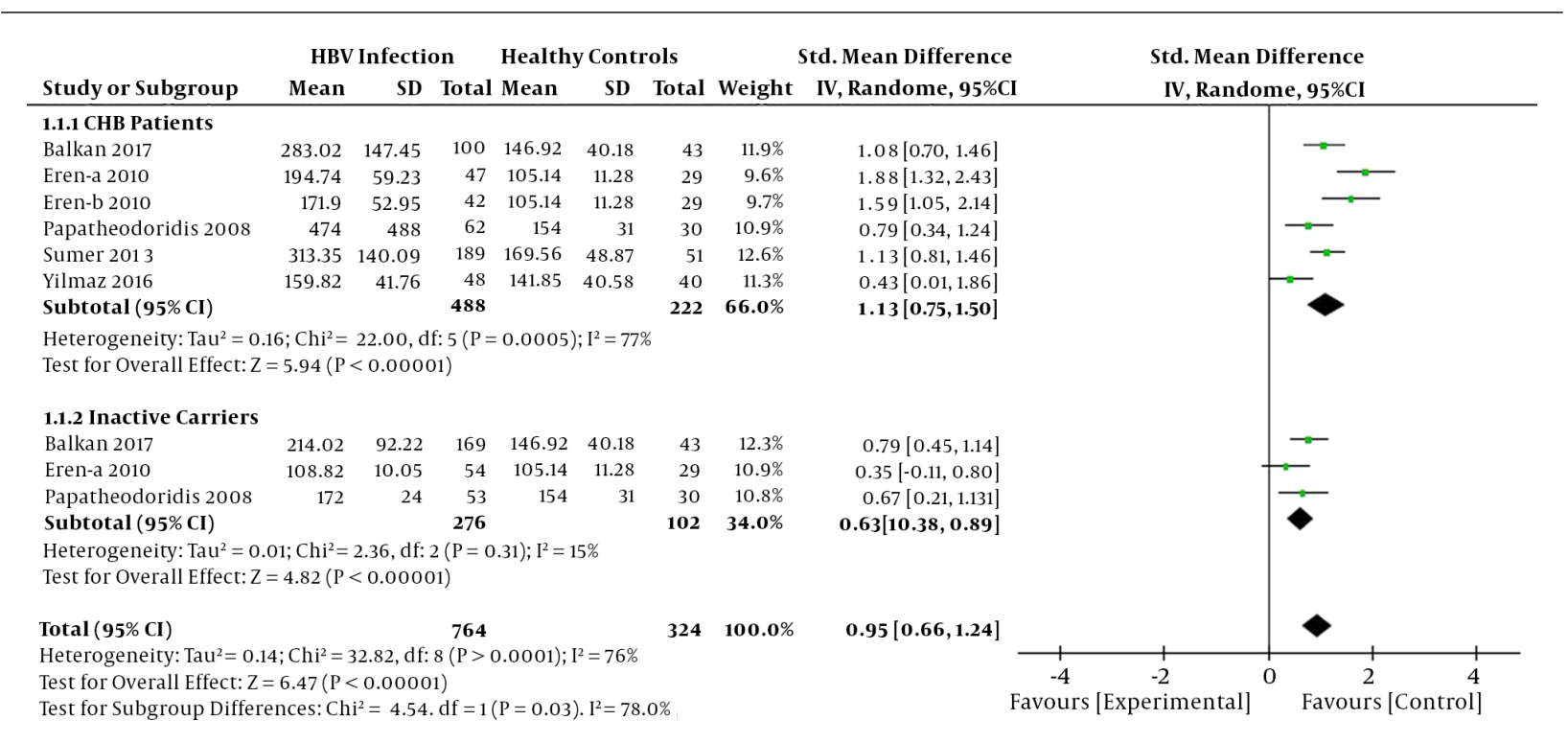

Figure 2. Forest plot of the differences of the elevated serum CK-18 M30 levels between chronic HBV infected patients and healthy controls

\begin{tabular}{|c|c|c|c|c|c|c|c|c|c|c|}
\hline \multirow{2}{*}{$\begin{array}{l}\text { Study or Subgroup } \\
\text { Balkan } 2017\end{array}$} & \multicolumn{3}{|c|}{ CHB Patients } & \multicolumn{3}{|c|}{ Inactive Carriers } & \multicolumn{2}{|c|}{$\begin{array}{r}\text { Std. Mean Difference } \\
\text { Weight } \quad \text { IV, Randome, 95\%CI } \\
\end{array}$} & \multicolumn{2}{|c|}{$\begin{array}{l}\text { Std. Mean Difference } \\
\text { IV, Randome, } 95 \% \mathrm{CI}\end{array}$} \\
\hline & 283.02 & 147.45 & 100 & 214.02 & 92.22 & 169 & $26.4 \%$ & $0.59[0.34,0.85]$ & & - \\
\hline Eren-a 2010 & 194.74 & 59.23 & 47 & 108.82 & 10.05 & 54 & $24.1 \%$ & $2.08[1.59,2.57]$ & & -- \\
\hline Eren-b 2010 & 171.9 & 52.95 & 42 & 108.82 & 10.05 & 54 & $24.2 \%$ & $1.75[1.27,2.22]$ & & $-m-$ \\
\hline Papatheodoridis 2008 & 474 & 488 & 62 & 172 & 24 & 53 & $25.3 \%$ & $0.84[0.45,1.22]$ & & - 믐 \\
\hline Total $(95 \% \mathrm{CI})$ & & & 251 & & & 3301 & $100.0 \%$ & $1.29[0.60,1.98]$ & & \\
\hline \multicolumn{9}{|c|}{$\begin{array}{l}\text { Heterogeneity: } \mathrm{Tau}^{2}=0.45 ; \mathrm{Chi}^{2}=38.87, \mathrm{df}=3(\mathrm{P}>0.0001) ; \mathrm{I}^{2}=92 \% \\
\text { Test for Overall Effect: } \mathrm{Z}=3.66(\mathrm{P}=\mathbf{0 . 0 0 0 2})\end{array}$} & $\begin{array}{ccc}-4 & -2 & 0 \\
\text { Favours [Experimental] }\end{array}$ & $\begin{array}{cc}2 & 4 \\
\text { Favours [Control] }\end{array}$ \\
\hline
\end{tabular}

Figure 3. Forest plot of the differences of the elevated serum CK-18 M30 levels between CHB patients and inactive carriers

mined through non-invasive diagnostic methods could be regarded as a useful biomarker for the diagnosis of chronic hepatitis $\mathrm{B}$.

Balkan et al. reported that the serum CK-18 M30 levels were significantly higher in asymptomatic HBV carriers $(198.77 \pm 77.62 \mathrm{U} / \mathrm{L})$ than in healthy controls (146.92 \pm 40.18 $\mathrm{U} / \mathrm{L})$, and the results suggested that $262 \mathrm{U} / \mathrm{L}$ cut-off value of CK-18 M30 possessed $85 \%$ specificity and $48 \%$ sensitivity to distinguish HBeAg-negative CHB from asymptomatic HBV carriers (27). We analyzed the difference of serum CK-18 M30 levels between inactive carriers and healthy controls in this study; a markedly elevated serum CK-18 M30 level was observed in the inactive carriers compared to healthy controls (SMD = 0.63, 95\% CI: $0.38-0.89, \mathrm{P}<0.001$ ). Sensitivity and publication bias analysis verified the accuracy of this analysis. We tried to interpret the phenomenon with some explanation in our meta-analysis. One possible reason is that most inactive carriers might have liver inflammation actually, while a normal ALT level was ob- served for more than 6 months during the diagnosis; therefore, the follow-up liver biopsy was quitted. Oliveira et al. carried out an investigation of the liver injury in patients presenting $\mathrm{HBsAg}(+)$ for more than six months, Anti$\mathrm{HBe}(+) / \mathrm{HBeAg}(-)$, viral load below 2,000 IU/mL, and serum ALT levels less than twice the upper limit of normality. The results showed that 11/27 (40.7\%) patients actually had advanced liver injury (35). Besides, research has shown that almost $44 \%$ of $\operatorname{HBsAg}(-)$ hepatitis patients have a normal range of ALT during the majority period despite having advanced liver diseases (34).

Our study first performed a systematic review of all eligible English publications about the association between serum CK-18 M30 levels and chronic HBV infection. Although the effort was made to conduct a comprehensive analysis, some limitations still should be acknowledged. First, the number of eligible studies and the sample size of the studies were relatively small, which limited the statistical power of our analysis, and might be a main reason for 

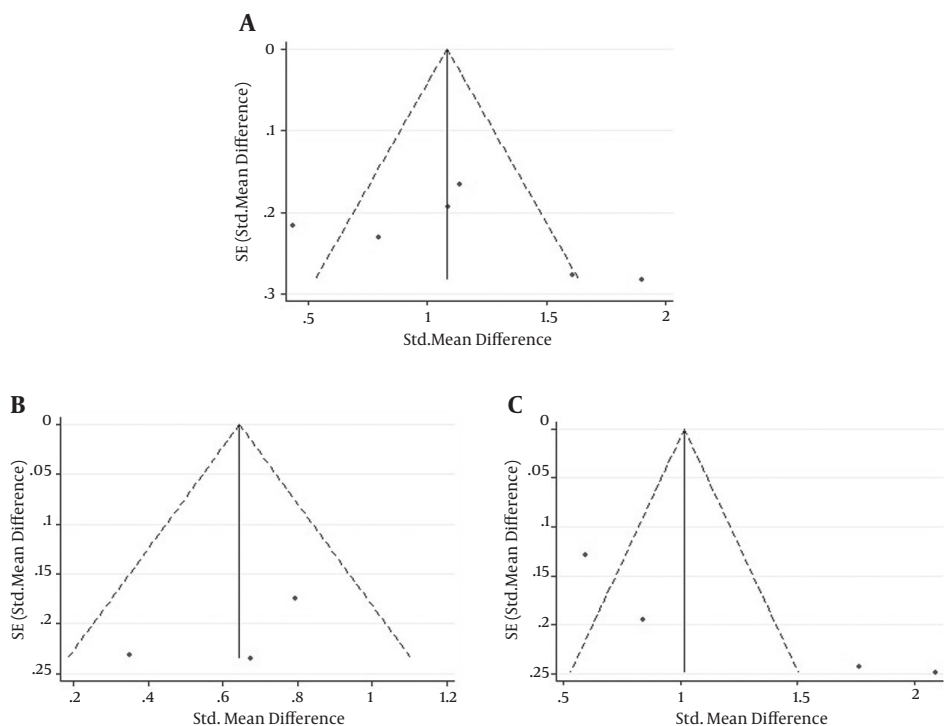

Figure 4. Funnel plot of publication bias on the difference of elevated serum CK-18 M30 levels between A, chronic HBV infected patients and healthy controls; B, inactive carriers and healthy controls; C, CHB patients and inactive carriers. SE: standard error.

the heterogeneity. Second, although no publication bias was observed in this meta-analysis, the results may not be exact due to the low number of studies. Third, the metaanalysis included English-language studies and grey literature or unpublished studies were not searched. Finally, we did not conduct any meta-regression analysis since the number of included studies was low.

In conclusion, this meta-analysis provides a strong evidence for the association of serum CK-18 M30 levels with the severity of liver injury in chronic hepatitis B patients. The CK-18 M30 levels could be adopted as a useful noninvasive biomarker for the diagnosis of chronic HBV infection. Further studies with larger populations and a comprehensive design are needed to investigate the diagnostic and physiotherapeutic value of CK-18 M30 for chronic hepatitis B patients.

\section{Acknowledgments}

This study was supported by grants from national natural science foundation of China (31770837).

\section{Footnote}

Authors' Contribution: Changfei Li and Shousheng Liu contributed equally to this work. Study concept and design: Changfei Li and Shousheng Liu; articles search and data extraction: Changfei Li and Shousheng Liu; analysis and interpretation of data: Changfei Li, Linlin Lu, and Quanjiang Dong; drafting of the manuscript: Changfei Li,
Shousheng Liu, and Yongning Xin; critical revision of the manuscript for important intellectual content: Yongning Xin and Shiying Xuan; study supervision: Shiying Xuan.

\section{References}

1. Ganem D, Prince AM. Hepatitis B virus infection-natural history and clinical consequences. $N$ Engl J Med. 2004;350(11):1118-29. doi: 10.1056/NEJMra031087. [PubMed: 15014185].

2. Zamor PJ, delemos AS, Russo MW. Viral hepatitis and hepatocellular carcinoma: etiology and management. J Gastrointest Oncol. 2017;8(2):229-42. doi:10.21037/jgo.2017.03.14. [PubMed: 28480063].

3. Bitton Alaluf M, Shlomai A. New therapies for chronic hepatitis B. Liver Int. 2016;36(6):775-82. doi: 10.1111/liv.13086. [PubMed: 26854115].

4. Kang SM, Shin MJ, Kim JH, Oh JW. Proteomic profiling of cellular proteins interacting with the hepatitis $\mathrm{C}$ virus core protein. Proteomics. 2005;5(8):2227-37. doi: 10.1002/pmic.200401093. [PubMed: 15846844].

5. Zeng DW, Dong J, Liu YR, Jiang JJ, Zhu YY. Noninvasive models for assessment of liver fibrosis in patients with chronic hepatitis B virus infection. World J Gastroenterol. 2016;22(29):6663-72. doi: 10.3748/wjg.v22.i29.6663. [PubMed: 27547009].

6. Invernizzi F, Vigano M, Grossi G, Lampertico P. The prognosis and management of inactive HBV carriers. Liver Int. 2016;36 Suppl 1:100-4. doi: 10.1111/liv.13006. [PubMed: 26725905].

7. Branchi F, Conti CB, Baccarin A, Lampertico P, Conte D, Fraquelli M. Non-invasive assessment of liver fibrosis in chronic hepatitis B. World J Gastroenterol. 2014;20(40):14568-80. doi: 10.3748/wjg.v20.i40.14568. [PubMed: 25356021].

8. Pan CQ, Zhang JX. Natural History and Clinical Consequences of Hepatitis B Virus Infection. Int J Med Sci. 2005;2(1):36-40. [PubMed: 15968338].

9. Jieanu CF, Ungureanu BS, Sandulescu DL, Gheonea IA, Tudorascu DR, Ciurea ME, et al. Quantification of liver fibrosis in chronic hepatitis B virus infection. J Med Life. 2015;8(3):285-90. [PubMed: 26351528] 
10. Bedossa P, Carrat F. Liver biopsy: the best, not the gold standard. J Hepatol. 2009;50(1):1-3. doi: 10.1016/j.jhep.2008.10.014. [PubMed: 19017551].

11. Bedossa P, Dargere D, Paradis V. Sampling variability of liver fibrosis in chronic hepatitis C. Hepatology. 2003;38(6):1449-57. doi: 10.1016/j.hep.2003.09.022. [PubMed:14647056].

12. Ijaz B, Ahmad W, Javed FT, Gull S, Hassan S. Revised cutoff values of ALT and HBV DNA level can better differentiate HBeAg (-) chronic inactive HBV patients from active carriers. Virol J. 2011;8:86. doi: 10.1186/1743422X-8-86. [PubMed: 21352567].

13. Wai CT, Greenson JK, Fontana RJ, Kalbfleisch JD, Marrero JA, Conjeevaram HS, et al. A simple noninvasive index can predict both significant fibrosis and cirrhosis in patients with chronic hepatitis C. Hepatology. 2003;38(2):518-26. doi: 10.1053/jhep.2003.50346. [PubMed: 12883497].

14. Fitzpatrick E, Mitry RR, Quaglia A, Hussain MJ, DeBruyne R, Dhawan A. Serum levels of CK18 M30 and leptin are useful predictors of steatohepatitis and fibrosis in paediatric NAFLD. J Pediatr Gastroenterol Nutr. 2010;51(4):500-6. doi: 10.1097/MPG.0b013e3181e376be. [PubMed: 20808246].

15. Parikh P, Ryan JD, Tsochatzis EA. Fibrosis assessment in patients with chronic hepatitis B virus (HBV) infection. Ann Transl Med. 2017;5(3):40. doi: 10.21037/atm.2017.01.28. [PubMed: 28251119].

16. Lee JE, Lee JM, Lee KB, Yoon JH, Shin CI, Han JK, et al. Noninvasive assessment of hepatic fibrosis in patients with chronic hepatitis B viral infection using magnetic resonance elastography. Korean J Radiol. 2014;15(2):210-7. doi: 10.3348/kjr.2014.15.2.210. [PubMed: 24643284].

17. Eren F, Yilmaz Y, Kose S, Ozdemir FT, Yonal O, Kurt R, et al. Caspase-cleaved fragments of cytokeratin 18 in patients with chronic hepatitis B. Clin Chim Acta. 2010;411(23-24):2029-32. doi: 10.1016/j.cca.2010.08.035. [PubMed: 20807521].

18. Cave M, Falkner KC, Henry L, Costello B, Gregory B, McClain CJ. Serum cytokeratin 18 and cytokine elevations suggest a high prevalence of occupational liver disease in highly exposed elastomer/polymer workers. J Occup Environ Med. 2011;53(10):1128-33. doi: 10.1097/JOM.0b013e31822cfd68. [PubMed: 21915069].

19. Rosso C, Caviglia GP, Abate ML, Vanni E, Mezzabotta L, Touscoz GA, et al. Cytokeratin 18-Aspartate396 apoptotic fragment for fibrosis detection in patients with non-alcoholic fatty liver disease and chronic viral hepatitis. Dig Liver Dis. 2016;48(1):55-61. doi: 10.1016/j.dld.2015.09.008. [PubMed: 26514735].

20. Yang ZH, Yang SX, Qin CZ, Chen YX. Clinical values of elevated serum cytokeratin-18 levels in hepatitis: a meta-analysis. Hepat Mon. 2015;15(5). e25328. doi: 10.5812/hepatmon.15(5)2015.25328. [PubMed: 26045704].

21. Feldstein AE, Alkhouri N, De Vito R, Alisi A, Lopez R, Nobili V. Serum cytokeratin-18 fragment levels are useful biomarkers for nonalcoholic steatohepatitis in children. Am J Gastroenterol. 2013;108(9):152631. doi: 10.1038/ajg.2013.168. [PubMed: 23752877].

22. Liang J, Liu F, Wang F, Han T, Jing L, Ma Z, et al. A Noninvasive Score Model for Prediction of NASH in Patients with Chronic Hepatitis B and Nonalcoholic Fatty Liver Disease. Biomed Res Int. 2017;2017:8793278. doi: 10.1155/2017/8793278. [PubMed: 28349067].

23. Shi J, Ying H, Du J, Shen B. Serum Sclerostin Levels in Patients with Ankylosing Spondylitis and Rheumatoid Arthritis: A Systematic Review and Meta-Analysis. Biomed Res Int. 2017;2017:9295313. doi: 10.1155/2017/9295313. [PubMed: 28553652].

24. Papatheodoridis GV, Hadziyannis E, Tsochatzis E, Chrysanthos N, Georgiou A, Kafiri G, et al. Serum apoptotic caspase activity as a marker of severity in HBeAg-negative chronic hepatitis B virus infection. Gut. 2008;57(4):500-6. doi: 10.1136/gut.2007.123943. [PubMed: 18025069].

25. Sumer S, Aktug Demir N, Kolgelier S, Cagkan Inkaya A, Arpaci A, Saltuk Demir L, et al. The Clinical Significance of Serum Apoptotic Cytokeratin 18 Neoepitope M30 (CK-18 M30) and Matrix Metalloproteinase 2 (MMP-2) Levels in Chronic Hepatitis B Patients with Cirrhosis. Hepat Mon. 2013;13(6). e10106. doi: 10.5812/hepatmon.10106. [PubMed: 24032040].

26. Yilmaz B, Aktas B, Altinbas A, Ginis Z, Ozturk G, Ekiz F, et al. The Role of M30 in Predicting the Severity of Liver Fibrosis and Inflammation in Chronic Hepatitis B Patients. Hepat Mon. 2016;16(9). e35640. doi: 10.5812/hepatmon.35640. [PubMed: 27822256].

27. Balkan A, Yilmaz N, Balkan Y, Koruk I, Orkmez M, Aydinli M, et al. Relationship between liver injury and serum cytokeratin 18 levels in asymptomatic hepatitis B virus carriers and in patients with chronic hepatitis B infection. Arab J Gastroenterol. 2017;18(2):98-103. doi: 10.1016/j.ajg.2017.05.008. [PubMed: 28579343].

28. Moll R, Franke WW, Schiller DL, Geiger B, Krepler R. The catalog of human cytokeratins: patterns of expression in normal epithelia, tumors and cultured cells. Cell.1982;31(1):11-24. [PubMed: 6186379].

29. Linder S, Olofsson MH, Herrmann R, Ulukaya E. Utilization of cytokeratin-based biomarkers for pharmacodynamic studies. Expert Rev Mol Diagn. 2010;10(3):353-9. doi: 10.1586/erm.10.14. [PubMed: 20370591].

30. Linder S, Havelka AM, Ueno T, Shoshan MC. Determining tumor apoptosis and necrosis in patient serum using cytokeratin 18 as a biomarker. Cancer Lett. 2004;214(1):1-9. doi: 10.1016/j.canlet.2004.06.032. [PubMed: 15331168].

31. Feldstein AE, Wieckowska A, Lopez AR, Liu YC, Zein NN, McCullough AJ. Cytokeratin-18 fragment levels as noninvasive biomarkers for nonalcoholic steatohepatitis: a multicenter validation study. Hepatology. 2009;50(4):1072-8. doi: 10.1002/hep.23050. [PubMed:19585618].

32. Aida Y, Abe H, Tomita Y, Nagano T, Seki N, Sugita T, et al. Serum cytokeratin 18 fragment level as a noninvasive biomarker for non-alcoholic fatty liver disease. Int J Clin Exp Med. 2014;7(11):4191-8. [PubMed: 25550930].

33. Chen J, Zhu Y, Zheng Q, Jiang J. Serum cytokeratin-18 in the diagnosis of non-alcoholic steatohepatitis: A meta-analysis. Hepatol Res. 2014;44(8):854-62. doi: 10.1111/hepr.12197. [PubMed: 23834322].

34. Swiderska M, Jaroszewicz J, Parfieniuk-Kowerda A, Rogalska-Plonska M, Stawicka A, Panasiuk A, et al. Serum Cytokeratin 18 M30 Levels in Chronic Hepatitis B Reflect Both Phase and Histological Activities of Disease. Mediators Inflamm. 2017;2017:3480234. doi: 10.1155/2017/3480234. [PubMed: 28827897].

35. Oliveira VO, Oliveira JP, Franca EV, Brito HL, Nascimento TV, Franca A. Advanced liver injury in patients with chronic hepatitis $b$ and viral load below 2,000 IU/mL. Rev Inst Med Trop Sao Paulo. 2016;58:65. doi: 10.1590/S1678-9946201658065. [PubMed: 27680170]. 